Carla Vitola Gonçalves ${ }^{1}$

Raul Mendoza Sassi ${ }^{2}$

Isabel Oliveira Netto ${ }^{3}$

Natália Bolbadilha de CAstro ${ }^{4}$

ANA PAULA BORTOLOMEDI ${ }^{4}$

\title{
Cobertura do citopatológico do colo uterino em Unidades Básicas de Saúde da Família
}

\section{CytopathologicalcoverageoffhecervixinBasicHealthUnitsoftheFamily}

Artigo original

Palavras-chave

Garantia da qualidade dos cuidados de saúde

Esfregaço vaginal

Colo do útero/citologia

Desigualdades em saúde

Saúde da mulher

Centros de Saúde

Keywords

Quality assurance, health care Vaginal smears

Cervix uteri/cytology

Health inequalities

Woman health

Health centers

\section{Resumo}

OBJETIVOS: Avaliar a cobertura do citopatológico em Unidades Básicas de Saúde da Família (UBSF) e descrever características da não realização deste exame nos últimos três anos. MÉTODOS: Estudo transversal, realizado em Rio Grande (RS), em áreas cobertas pelas Equipes de Saúde da Família (ESF). As entrevistas foram realizadas no domicílio das mulheres, pelos alunos participantes do PET-Saúde. A análise bruta utilizou o software SPSS, onde foram calculados a razão de prevalência, intervalos de confiança de $95 \%$ e valor p. A análise multivariada foi realizada por regressão de Poisson, no programa Stata 9.0, onde ingressaram as variáveis com valor p de até 0,20 na análise bruta. No primeiro nível, temos as variáveis idade, ter companheiro e saber ler e escrever. No segundo nível, ingressaram as variáveis número de consultas e oferecimento da coleta de citopatológico. RESULTADOS: A prevalência de citopatológico realizado há 36 meses ou menos foi de 66,3\%. Na análise ajustada, as mulheres com idade de 19 anos ou menos $(p<0,001)$, sem companheiro $(p<0,001)$, que não sabiam ler e escrever $(p=0,01)$, que nunca consultaram na unidade básica $(p=0,02)$ e que o exame não havia sido oferecido na consulta $(p=0,006)$, apresentaram maior probabilidade não ter o seu exame de citopatológico coletado nos últimos 36 meses. CONCLUSÃO: $\bigcirc$ serviço local de saúde mostrou-se pouco efetivo e desigual. Pouco efetivo porque cobriu menor número de mulheres do que o indicado pela Organização Mundial da Saúde e desigual porque o acesso a esse exame variou conforme algumas características das usuárias.

Abstract

PURPOSE: To evaluate the coverage of Pap smear cytology at Basic Family Health Units (BFHU) and to describe the characteristics of non-performance of this test in the last three years. METHODS: A cross-sectional study was conducted in Rio Grande (RS), Brazil, in areas covered by the Family Health Teams Family (FHT). The interviews were conducted by students participating in the Health-PET, at women's home. Crude analysis was performed using SPSS software to calculate prevalence ratio, 95\% confidence intervals and $p$ value. Multivariate analysis was performed by Poisson regression using Stata 9.0 software, which were included the variables with p value of up to 0.20 in the crude analysis. At the first level, the variables were age, having a partner, and literacy. At the second level, the variables were number of visits and offer of a Pap smear. RESULTS: The prevalence of Pap cytology performed 36 months ago or less was $66.3 \%$. In adjusted analysis, women aged 19 years or less $(p<0.001)$, without a partner $(p<0.001)$, illiterate $(p=0.01)$, who had never consulted at the basic unit $(p=0.02)$ and who had not been offered the examination during the visit $(p=0.006)$, were more likely not to have had a cytopathology exam in the last 36 months. CONCLUSION: The local health proved to be ineffective and inequitable. Ineffective because it covers fewer women than indicated by the World Health Organization and uneven because access to this test varied according to some characteristics of the users.
Correspondência:

Carla Vitola Gonçalves Faculdade de Medicina da Universidade Federal do Rio Grande Rua General Osório, $s / n-4^{\circ}$ andar Centro - CEP: $96201-900-$ Rio Grande (RS), Brasil

Recebido 02/08/2011

Aceito com modificacọoes

20/09/2011
Faculdade de Medicina da Universidade Federal do Rio Grande - FURG - Rio Grande (RS), Brasil.

' Professora Adjunta de Ginecologia e Obstetrícia da Faculdade de Medicina da Universidade Federal do Rio Grande - FURG - Rio Grande (RS), Brasil.

2 Professor Associado de Medicina Social da Faculdade de Medicina da Universidade Federal do Rio Grande - FURG - Rio Grande (RS), Brasil.

${ }_{3}^{3}$ Professora Associada de Clínica Médica da Faculdade de Medicina da Universidade Federal do Rio Grande - FURG - Rio Grande (RS), Brasil.

${ }^{4}$ Acadêmicas do curso de Medicina da Universidade Federal do Rio Grande - FURG - Rio Grande (RS), Brasil. 


\section{Introdução}

O câncer do colo do útero é o segundo tipo de câncer mais frequente entre as mulheres no mundo, com aproximadamente 500 mil casos novos por ano, sendo responsável pelo óbito de 230 mil mulheres por ano ${ }^{1}$. Sua incidência é cerca de duas vezes mais elevada em países em desenvolvimento quando comparada com países desenvolvidos. No Brasil, o Instituto Nacional do Câncer (INCA) estimou que o número de casos novos de câncer do colo do útero para o Brasil no ano de 2010 foi de 18.430 , com um risco estimado de 18 casos a cada 100 mil mulheres. Nesse mesmo ano, o estado do Rio Grande do Sul teve uma taxa estimada de 21,53 casos para cada 100 mil habitantes ${ }^{2}$.

A realização periódica do exame citopatológico continua sendo a estratégia mais adotada para o rastreamento do câncer do colo uterino ${ }^{1-3}$. Embora o exame de citologia cervical tenha sido introduzido no Brasil na década de 1950, a cobertura nacional deste exame ainda encontra-se abaixo do preconizado pela Organização Mundial da Saúde $(\mathrm{OMS})^{1-5}$. Dois estudos de abrangência nacional, onde foram entrevistadas 5.000 brasileiras com idade acima de 18 anos mostraram uma cobertura estimada do exame de citologia cervical nos últimos 3 anos entre 66 e $68 \%$ 4,5.

Quando se busca analisar as características associadas a não atualização do exame citopatológico, observa-se que os fatores que levam a não realização deste exame têm se repetido nos diferentes estudos brasileiros. Entre eles estão: mulheres pertencentes às faixas etárias mais jovens, não brancas, com baixo nível socioeconômico, com baixa escolaridade, sem companheiro e que não consultaram no último ano ${ }^{4-10}$.

O objetivo deste estudo foi avaliar a cobertura do exame citopatológico do colo uterino na rede básica de saúde da família da cidade do Rio Grande (RS), assim como descrever características associadas a não realização desse exame.

\section{Métodos}

Este estudo foi realizado no município do Rio Grande, de porte médio, com cerca de 197.228 habitantes e que está localizado na planície costeira sul do estado do Rio Grande do Sul. Sua economia é bastante diversificada e o seu produto interno bruto (PIB) per capitalano é de aproximadamente $\mathrm{R} \$ 27.624,00^{11}$.

O delineamento utilizado para o estudo foi do tipo transversal de base populacional, adequado para realizar diagnósticos de situação de saúde e avaliação de serviços. A população alvo eram mulheres de 14 anos ou mais residentes em áreas cobertas pelas Equipes de Saúde da Família (ESF) de Rio Grande. A ESF está organizada em áreas, onde vivem em torno de $800 \mathrm{a}$ 1.000 famílias. Cada área é dividida em seis microáreas, sendo cada microárea atendida por um agente comunitário de saúde (ACS) e tendo como sede a Unidade Básica de Saúde (UBS).
Nessa UBS trabalha uma equipe multiprofissional formada, além dos ACS, por um(a) médico(a), um(a) enfermeiro(a), dois(as) técnicos(as) de enfermagem e um(a) recepcionista. Os prontuários são organizados por família e cada uma é identificada por um número, correspondente à microárea e à família. As Unidades Básicas de Saúde da Família (UBSF) em Rio Grande totalizavam, no momento da pesquisa, 27. Para esta pesquisa, foram selecionadas seis UBSF onde se desenvolvia o Programa de Educação pelo Trabalho para a Saúde da Faculdade de Medicina da Universidade Federal do Rio Grande (PET-Saúde).

Para fins de cálculo da amostra, identificou-se que a menor prevalência esperada entre os desfechos de interesse situava-se em $5 \%$. Assim, optou-se por calcular uma amostra que estimasse uma prevalência com esse valor esperado, uma precisão de $\pm 2 \%$, e um nível de confiança de $95 \%$. O tamanho da amostra, com esses parâmetros, deveria incluir 502 mulheres, já acrescidos de $10 \%$ para possíveis perdas. O processo de amostragem teve três estágios. No primeiro estágio, foi realizada uma amostragem de tipo proporcional considerando as percentagens de mulheres com 14 anos ou mais cadastradas em cada uma das áreas do estudo. $\mathrm{Na}$ segunda etapa, o tamanho de amostra, proporcional para cada área, foi dividido pelas microáreas. Finalizando, em cada microárea foi realizado um sorteio aleatório simples, utilizando-se uma tabela de números construída para essa finalidade. O número de cada família na microárea serviu para identificar a família sorteada.

Existiam em média 900 domicílios por área, totalizando, nas 6 áreas, 5.400. Estimou-se 1,2 mulheres por domicílio. Assim, nas seis áreas, foi visitado um total de 418 domicílios para identificar as mulheres. Preferiu-se essa estratégia em estágios (com visitas a domicílios para identificar mulheres) ao sorteio aleatório simples das mulheres dos domicílios já identificados e cadastrados pelos ACS, com a finalidade de evitar um viés de seleção, uma vez que seria possível que algum sujeito da pesquisa não estivesse identificado e cadastrado ainda.

Após o sorteio e a identificação dos domicílios que deveriam ser visitados em cada microárea, uma dupla de entrevistadores, acompanhada pelo ACS responsável pela microárea, se deslocava até os domicílios para realizar as entrevistas. Após a apresentação da família aos entrevistadores, foi solicitado que o ACS não estivesse presente no momento da entrevista. Essa medida foi tomada para evitar algum viés de informação em função da presença do responsável pelo acompanhamento da família.

As entrevistas foram realizadas no período entre outubro de 2009 e fevereiro de 2010 pelos alunos participantes do PET-Saúde, os quais receberam, para essa pesquisa, uma capacitação específica constituída de apresentação e leitura dos instrumentos, aplicação do questionário entre dois entrevistadores e dramatização de situações com representação dos atores 
envolvidos nas entrevistas. Após o treinamento, foi realizado um estudo piloto com a finalidade de testar os instrumentos e a logística planejada. Para isso, foram visitados domicílios, entre aqueles que não foram sorteados, por uma dupla de entrevistadores. Sendo necessárias mudanças na abordagem da questão sobre a realização do exame de pré-câncer, sendo incluídos os termos citopatológico e Papanicolaou.

A variável dependente do estudo era adequação do exame citopatológico $(\mathrm{CP})$, definida como a proporção de mulheres que realizaram o $\mathrm{CP}$ segundo as recomendações do Ministério da Saúde (36 meses ou menos). Entre as variáveis independentes estudadas estavam: idade, cor da pele, estado civil, escolaridade, renda familiar, realização de consulta nos últimos dois meses e local onde costuma consultar.

Os dados foram digitados em banco construído no Epi Info 6.04. Procedeu-se à dupla digitação e posterior limpeza de dados identificando e corrigindo erros de amplitude e/ou consistência. As questões abertas foram codificadas posteriormente, categorizando segundo as respostas mais frequentes. A análise bruta utilizou os programas do software SPSS, onde foram calculados a razão de prevalência, intervalos de confiança de $95 \%$ e valor p. A análise multivariada foi realizada através da regressão de Poisson, pela possibilidade de demonstrar a magnitude das associações sem problemas de estimativa, oriundas de razões de diferenças. No programa Stata 9.0, foi seguido um modelo hierarquizado de análise, no qual ingressaram as variáveis com valor $\mathrm{p}$ de até 0,20 na análise bruta. No primeiro nível, ingressaram as variáveis idade, ter companheiro e saber ler e escrever. No segundo nível, ingressaram no modelo as variáveis que representavam o atendimento nas UBSF, como número de consultas e oferecimento da coleta de citopatológico. Em cada nível do modelo, foram retiradas as variáveis que não obtiveram valor $\mathrm{p}$ menor que 0,05 .

Em relação às mulheres incluídas, $45 \%$ apresentavam idade entre 20 e 39 anos, $65 \%$ eram de cor branca e $61,5 \%$ tinham companheiro no momento da entrevista. Quanto à escolaridade, $89,6 \%$ das mulheres sabiam ler e escrever, sendo que, $65 \%$ dessas haviam estudado durante cinco anos ou mais.

\section{Resultados}

Dentre as 454 mulheres incluídas nesse estudo, 98\% delas já tinham ouvido falar do exame citopatológico do colo do útero, porém apenas $76 \%$ tinham feito $\mathrm{CP}$ alguma vez na vida. Além disso, somente $66,3 \%$ haviam sido submetidas ao exame de CP há 36 meses ou menos.

$\mathrm{Na}$ análise do serviço público, $72 \%$ mencionaram a existência do atendimento pelo PSF no bairro de moradia. Do total de mulheres entrevistadas, $41,4 \%$ referiram costumar consultar no posto de saúde, sendo que $36,8 \%$ compareceram a pelo menos 1 a 4 consultas nos 2 meses anteriores à entrevista. Somado a isso, a coleta do exame de
CP foi oferecida na UBS para $60,8 \%$ das mulheres, porém apenas $49,1 \%$ foram submetidas ao exame na UBS.

$\mathrm{Na}$ Tabela 1, temos a análise das variáveis demográficas e socioeconômicas, sendo possível constatar que os extremos de idade, ou seja, mulheres com 19 anos ou menos e aquelas com 60 anos ou mais, mostraram maior prevalência de citologia desatualizada, quando comparadas às faixas etárias intermediárias $(\mathrm{p}<0,0001)$. As mulheres brancas não tiveram maior prevalência de citologia cervical atualizada quando comparadas as não brancas, já que revelaram proporções semelhantes de citologia não atualizada. As pacientes sem companheiro no momento da entrevista apresentaram menor cobertura do exame de $\mathrm{CP}$ do que as pacientes com companheiro (IC95\% $=1,7-2,9$ e $\mathrm{p}<0,001)$.

Em relação à escolaridade, foi demonstrada uma probabilidade de $48 \%$ das mulheres que sabiam ler e escrever de ter o seu $\mathrm{CP}$ atualizado, quando comparadas às que não liam e escreviam ( $\mathrm{RP}=0,6$ com IC95\%=0,4-0,8). Embora não tenha sido registrada diferença significativa quanto ao tempo em anos de estudo, é possível observar que as

Tabela 1. Análise da cobertura e de fatores associados a não realização do citopatológico do colo uterino nos últimos 36 meses, em Unidades Básicas de Saúde da Família, em município do Rio Grande do Sul

\begin{tabular}{|c|c|c|c|c|c|}
\hline Variável & $\%$ & $\begin{array}{c}\text { Sem } \\
\text { citopatológico } \\
(\%)\end{array}$ & $\begin{array}{l}\text { Razão de } \\
\text { prevalência }\end{array}$ & IC95\% & $\begin{array}{c}\text { Valor } \\
\mathbf{p}\end{array}$ \\
\hline \multicolumn{6}{|l|}{ Idade (anos) } \\
\hline 19 ou menos & 9,7 & 77,3 & 1,0 & & $<0,001$ \\
\hline 20 a 39 & 44,9 & 25,0 & 0,3 & $0,2-0,4$ & \\
\hline 40 a 59 & 31,3 & 24,6 & 0,3 & $0,2-0,4$ & \\
\hline 60 ou mais & 14,1 & 51,6 & 0,6 & $0,5-0,8$ & \\
\hline \multicolumn{6}{|l|}{ Tem companheiro } \\
\hline $\operatorname{Sim}$ & 61,5 & 22,9 & 1,0 & & $<0,001$ \\
\hline Não & 38,5 & 50,9 & 2,2 & $1,7-2,9$ & \\
\hline \multicolumn{6}{|l|}{ Sabe ler e escrever } \\
\hline Não & 10,4 & 51,1 & 1,0 & & 0,008 \\
\hline $\operatorname{Sim}$ & 89,6 & 31,7 & 0,6 & $0,5-0,9$ & \\
\hline \multicolumn{6}{|l|}{ Renda per capita } \\
\hline <l salário-mínimo & 79,4 & 20,6 & 1,0 & & 0,2 \\
\hline 21 salário-mínimo & 35,4 & 28,6 & 0,8 & $0,6-1,1$ & \\
\hline \multicolumn{6}{|c|}{ Tem Programa de Saúde da Família } \\
\hline Não & 19,4 & 40,9 & 1,0 & & 0,1 \\
\hline Não sabe & 8,6 & 41,0 & 1,0 & $0,7-1,6$ & \\
\hline $\operatorname{Sim}$ & 72 & 30,9 & 0,8 & $0,6-1,0$ & \\
\hline \multicolumn{6}{|l|}{ Número de consultas } \\
\hline Nunca consultou & 58,6 & 39,8 & 1,0 & & 0,004 \\
\hline 1 a 4 & 36,8 & 24,6 & 0,6 & $0,5-0,8$ & \\
\hline 5 ou mais & 4,6 & 28,6 & 0,7 & $0,4-1,4$ & \\
\hline \multicolumn{6}{|c|}{ Foi oferecida coleta de citopatológico } \\
\hline Não & 39,2 & 47,8 & 1,0 & & $<0,001$ \\
\hline Sim & 60,8 & 24,6 & 0,5 & & \\
\hline
\end{tabular}

IC95\%: intervalos de confiança de $95 \%$. 
mulheres com nove ou mais anos de escolaridade tivessem seu CP atualizado. Quanto à renda em salário-mínimo, tanto a dividida em renda per capita como em quartil, não foi observada relevância estatística em relação à realização do CP nos últimos 36 meses (Tabela 1).

Levando em consideração as características dos serviços públicos prestados, o fato de a paciente saber se tem ou não PSF no seu bairro não demonstrou diferença significante para a atualização do exame de CP (Tabela 1). No entanto, as mulheres que nunca haviam consultado, apresentaram maior probabilidade de não realização do exame citopatológico $(\mathrm{p}=0,004)$, quando comparadas às que haviam consultado nos dois meses anteriores na UBS. Apesar disso, o número de consultas na UBS, nos últimos dois meses, não demonstrou significância estatística para uma maior cobertura do exame de CP (Tabela1). Quanto à oferta do citopatológico pelos profissionais de saúde das UBS, observa-se que o grupo de pacientes para as quais foi oferecida a coleta do exame de CP mostrou uma probabilidade de $49 \%$ de estar com seu exame atualizado (IC95\%=0,4-0,7 e p <0,001).

$\mathrm{Na}$ análise multivariada, quando incluímos no primeiro nível idade, presença de companheiro e saber ler e escrever, observamos que as mulheres com 19 anos ou menos, que não tinham companheiro, e que não sabiam ler e escrever apresentavam uma maior probabilidade não ter o seu exame de citopatológico coletado nos últimos 36 meses. No segundo nível da análise multivariada, foram incluídas as variáveis referentes ao número de consultas e ao oferecimento do CP na UBS, sendo constatado que as mulheres que nunca consultaram e aquelas em que não foi oportunizada a coleta do $\mathrm{CP}$, apresentam maior probabilidade de estarem com este exame desatualizado (Tabela 2).

Tabela 2. Análise ajustada para fatores de confusão associados a não realização do citopatológico do colo uterino nos últimos 36 meses, em Unidades Básicas de Saúde da Família, em município do Rio Grande do Sul

\begin{tabular}{|c|c|c|c|}
\hline Variável & $\begin{array}{l}\text { Razão de } \\
\text { prevalência }\end{array}$ & IC95\% & Valor $p$ \\
\hline \multicolumn{4}{|l|}{ Idade (anos) ${ }^{a}$} \\
\hline 19 ou menos & 1,0 & & $<0,001$ \\
\hline 20 a 39 & 0,4 & $0,3-0,5$ & \\
\hline 40 a 59 & 0,4 & $0,3-0,6$ & \\
\hline 60 ou mais & 0,6 & $0,5-0,8$ & \\
\hline \multicolumn{4}{|l|}{ Tem companheiro" } \\
\hline Sim & 1,0 & & $<0,001$ \\
\hline Não & 1,7 & $1,2-2,2$ & \\
\hline \multicolumn{4}{|l|}{ Sabe ler e escrever ${ }^{a}$} \\
\hline Não & 1,0 & & 0,01 \\
\hline Sim & 0,7 & $0,5-0,9$ & \\
\hline \multicolumn{4}{|l|}{ Número de consultas ${ }^{b}$} \\
\hline Nunca consultou & 1,0 & & 0,02 \\
\hline 1 a 4 & 0,6 & $0,5-0,9$ & \\
\hline 5 ou mais & 0,8 & $0,4-1,8$ & \\
\hline \multicolumn{4}{|c|}{ Foi oferecida coleta de $\mathrm{CPb}$} \\
\hline Não & 1,0 & & 0,006 \\
\hline Sim & 0,6 & $0,5-0,9$ & \\
\hline
\end{tabular}

IC95\%: intervalos de confiança de 95\%; CP: citopatológico; ' primeiro nível da análise ajustada; 'bsegundo nível da análise ajustada.

\section{Discussão}

O câncer do colo uterino está incluído na lista de mortes evitáveis. Estudo realizado na Nova Zelândia concluiu que o óbito por câncer do colo do útero seria evitável pela prevenção primária, práticas educativas e orientação, em 30\%; pela prevenção secundária, detecção precoce por meio de rastreamento, em $50 \%$; e pela prevenção terciária, acesso ao tratamento oportuno, em $20 \%$ dos $\operatorname{casos}^{12}$.

Cobertura é um termo descrito como a proporção da população-alvo que se beneficia de determinada intervenção ${ }^{13,14}$. Dentre as recomendações da OMS, para impactar o perfil epidemiológico do câncer do colo do útero tem-se de garantir uma cobertura de rastreamento mínima de $80 \%$ da população feminina de 25-59 anos, prezar pela qualidade de realização do exame citológico e garantir tratamento oportuno e seguimento das pacientes ${ }^{1,14}$. No entanto, entre as mulheres incluídas neste estudo, a cobertura do exame citopatológico do colo uterino nos últimos 3 anos foi de 66,3\%. Este resultado possui semelhança com os dados obtidos no Inquérito Populacional Nacional ${ }^{4}$ realizado no ano de 2003, que registrou cobertura de 65,5\% para mulheres com idade entre 18 e 69 anos; e com o estudo de base populacional efetuado, no ano de 2006 em Pernambuco, em que 58,7\% das mulheres tinham sido submetidas ao citopatológico nos últimos 3 anos?.

Entretanto, quando comparado a outro inquérito nacional desenvolvido em 15 capitais brasileiras no período de 2002-2003, o qual revelou coberturas entre 73,4\% em João Pessoa (PB) e 92,9\% em Vitória (ES), este estudo encontra-se com uma cobertura inferior à nacional ${ }^{10}$. Outras pesquisas realizadas na região sul também apresentaram prevalência de CP atualizado de $72 \%$ em Pelotas (RS $)^{15}$ e 85,5\% em São Leopoldo (RS) ${ }^{16}$.

Apesar da cobertura registrada neste estudo estar abaixo da preconizada pela OMS e da encontrada em outros estudos da região ${ }^{15,16}$, podemos observar uma melhora na prevalência de realização desse exame nesta cidade. Pesquisa realizada em 1995, mostrou que 57\% das mulheres riograndinas, em idade fértil, nunca haviam sido submetidas a um exame citopatológico do colo do útero ${ }^{6}$. Em 2007, um novo inquérito efetuado em gestantes, no mesmo município, apresentou uma cobertura de CP de $59,1 \%$, com 36,0\% das puérperas referindo nunca terem sido submetidas a coleta de citopatológico, deve-se levar em consideração que as gestantes apresentam maior probabilidade de estarem com seu exame citopatológico atualizado em relação às mulheres não grávidas, visto que nesse período os exames vaginais são mais frequentes para avaliar e tratar leucorréias ${ }^{7}$. Na pesquisa atual, apesar de 98\% da população estudada referir já ter ouvido falar 
do exame preventivo para o rastreamento de câncer do colo uterino, $34 \%$ das entrevistadas nunca haviam sido submetidas a esse exame.

Neste estudo, podemos observar que o exame é menos realizado em mulheres mais jovens e nas de maior idade. Vários trabalhos na literatura confirmaram que pacientes com idade inferior a 25 anos apresentam um maior risco de estarem com seu exame citopatológico não atualizado ${ }^{5,7,8}$. Entretanto, verifica-se que os achados de anormalidades citopatológicas em adolescentes sexualmente ativas têm aumentado progressivamente, alterando-se de $3 \%$, na década de 1970, para $20 \%$ na década de $1990^{2}$. Alguns autores referem que as adolescentes são mais suscetíveis à infecção pelo HPV e que as lesões precursoras do carcinoma cervical progridem mais rapidamente em mulheres jovens. Estes fatos estariam relacionados à imaturidade imunológica sistêmica e cervical, à maior exposição da zona escamo-colunar (JEC) e baixa incidência do uso de métodos de barreira ${ }^{1,17,18}$.

A associação da consulta ginecológica com problemas originados da vida sexual e para contracepção feita pela maioria das mulheres e não a uma perspectiva mais ampla de cuidado com a saúde, levaria mulheres sem companheiro a buscarem menos os serviços ginecológicos tendo, portanto, menor acesso ao exame $9,10,18,19$. As mulheres sem companheiro na época da entrevista apresentaram maior probabilidade de ficar com a sua citologia desatualizada, concordando com o observado por outros autores $5,6,9,15,16$.

É bem documentada na literatura científica que quanto menos tempo de estudo a mulher possui, menor é a probabilidade dela ser submetida ao exame citopatológico, talvez porque estas pacientes tenham um menor poder de pressão sobre os serviços de saúde na reivindicação de atendimento de qualidade $e^{6,9,20}$. Neste estudo, as mulheres que sabiam ler e escrever apresentaram maior probabilidade (33\%) de serem submetidas ao exame de citopatológico, do que as mulheres analfabetas.

Limitações no acesso aos serviços de saúde, por barreiras socioeconômicas, culturais, e geográficas se apresentam como responsáveis pela baixa cobertura dos exames de citologia oncótica do colo uterino ${ }^{20,21}$. Vários estudos associam a falta de consulta médica no último ano, como um fator preditivo a não realização do exame citopatológico nos últimos três anos ${ }^{2,8,22}$. Nesta pesquisa, as pacientes que nunca haviam consultado apresentaram maior probabilidade de não realização do exame citopatológico nos últimos três anos. Em relação ao número anual de consultas médicas, seria esperado que as mulheres com mais visitas se submetessem mais ao exame de CP. No entanto, constatou-se que o grupo de mulheres com mais de cinco consultas não apresentava maior prevalência de $\mathrm{CP}$ atualizado que o grupo que consultou uma a quatro vezes nos últimos dois meses. Além disso, o grupo de pacientes, para as quais não foi oferecida a coleta do exame pela rede de saúde, apresentou maior chance de estar com $\mathrm{CP}$ desatualizado. Este achado serve como alerta para o desperdício de oportunidades de prevenção.

A rede de saúde do município é abundante, metade das mulheres entrevistadas residirem a menos de um quilômetro do serviço de saúde e, segundo a coordenação local, o exame de citopatológico é oferecido em todos os postos do município ${ }^{6,7}$. Apesar das mulheres incluídas no estudo morarem em área de abrangência do PSF, a cobertura do exame citopatológico nesta população foi abaixo dos $80 \%$ recomendados pela $\mathrm{OMS}^{1}$. Estudo de base populacional realizado no estado de Pernambuco, no ano de 2006, encontrou resultados semelhantes ${ }^{9}$. Considerando a importância da estratégia de saúde da família na reorganização da atenção primária, com a premissa de funcionar como porta de entrada para o sistema público de saúde, é recomendável que os gestores intervenham, através de processos sistemáticos de monitoramento e avaliação, visando à melhoria da atenção à saúde prestada.

Quanto à prevalência da realização do citopatológico associado a fatores de barreira específicos, observou-se, após a análise ajustada, que a mulheres com idade igual ou menor a 19 anos, sem companheiro, que não sabiam ler e escrever, que nunca consultaram na UBSF e às quais não foi oferecido o exame de Papanicolaou na consulta médica, apresentaram maior prevalência de citologia cervical desatualizado. Revisão sistemática da literatura realizada em 2004, visando informações sobre a cobertura do exame Papanicolaou no Brasil, observou que os fatores que levam a não realização deste exame têm se repetido nos diferentes estudos. Entre eles estão: mulheres com baixo nível socioeconômico, baixa escolaridade e pertencentes às faixas etárias mais jovens 5 . Estudo transversal de base populacional realizado em Pelotas (2006) entrevistou 1.404 mulheres de 20 a 59 anos, mostrando diferença estatística significante associada a não realização do $\mathrm{CP}$ nos últimos 3 anos, em relação às seguintes variáveis: faixa etária jovem, mulheres não brancas, escolaridade menor ou igual a 4 anos e não apresentarem companheiro fixo ${ }^{23}$.

Concluímos que apesar do aumento da cobertura do exame citopatológico do colo uterino na cidade do Rio Grande nos últimos anos ${ }^{6-8,23}$, o serviço local de saúde local ainda mostra-se pouco efetivo e desigual, em relação à coleta do exame citopatológico. Pouco efetivo porque cobriu menor número de mulheres do que o indicado pela $\mathrm{OMS}^{1}$ e desigual porque o acesso a esse exame variou conforme algumas características das usuárias. Para contornar este quadro, Dias-da-Costa et al. ${ }^{24}$ realizaram auditorias em um posto de saúde da periferia de Pelotas (RS), com o intuito de avaliar a qualidade da atenção prestada nesta unidade. Esta atividade resultou em melhora objetiva dos serviços 
oferecidos às gestantes, entre eles o exame citopatológico, que apresentou prevalência de 4,3\% em 1994, passando a ser realizado em 69,3\% das gestantes em 1998.

Ao se interpretar esses dados, deve-se ter em mente algumas limitações que podem acometer os estudos transversais, que são o viés de memória e o de aferição. Neste estudo, a variável mais afetada pelo viés de memória foi a data do último exame ao qual a mulher foi submetida. O viés de aferição pode ter superestimado a sua realização e subestimado o tempo decorrido do último exame ${ }^{25}$. A variável cor da pele era avaliada pelo próprio entrevistador, podendo apresentar algum grau de subjetividade, uma vez que não mostrou significância estatística neste estudo.

\section{Referências}

1. World Health Organization. Comprehensive cervical cancer control: a guide to essential practice. Geneva: WHO; 2007.

2. Brasil. Ministério da Saúde. Instituto Nacional de Câncer. Estimativas da incidência e mortalidade por câncer. Rio de Janeiro: INCA; 2011 .

3. Brasil. Ministério da Saúde. Instituto Nacional de Câncer. Diretrizes Brasileiras para o Rastreamento do Câncer do Colo do Útero: atualização 2011. Rio de Janeiro: INCA; 2011

4. Novaes HMD, Braga $P E$, Schout D. Fatores associados à realização de exames preventivos para câncer nas mulheres brasileiras, PNAD 2003. Ciênc Saúde Coletiva. 2006; 11 (4):1023-35

5. Martins LFL, Thuler LCS, Valente JG. Cobertura do exame de Papanicolaou no Brasil e seu fatores determinantes: uma revisão sistemática da literatura. Rev Bras Ginecol Obstet. 2005;27(8):485-92.

6. Cesar JA, Horta BL, Gomes G, Houlthausen RS, Willrich RM, Kaercher $A$, et al. Fatores associados à não realização de exame citopatológico de colo uterino no sul do Brasil. Cad Saúde Pública. 2003; 19(5):1365-72.

7. Gonçalves CV, Duarte G, Costa JSD, Quintana SM, Marcolin AC Perdas de oportunidades na prevenção do câncer de colo uterino durante o pré-natal. Ciênc Saúde Coletiva. 2011;16(5):2501-10.

8. Quadros CAT, Victora CG, Costa JSD. Coverage and focus of a cervical cancer prevention program in southern Brazil. Rev Panam Salud Publica. 2004; 16(4):223-32.

9. Albuquerque KM, Frias PG, Andrade CLT, Aquino EML, Menezes G, Szwarcwald LC. Cobertura do teste de Papanicolaou e fatores associados à não-realização: um olhar sobre o Programa de Prevenção do Câncer do Colo do Útero em Pernambuco, Brasil. Cad Saúde Pública. 2009;25(Suppl 2):S301-9.

10. Szwarcwald CL, Viacava F, Vasconcellos MTL, Leal MC, Azevedo LO, Queiroz RSB, et al. Pesquisa Mundial de Saúde 2003: O Brasil em números. RADIS. 2004;(23):14-33.

11. Instituto Brasileiro de Geografia e Estatística [Internet]. Censo demográfico 2010 [citado 2011 Maio 5]. Disponível em: <http://www.ibge.gov. $\mathrm{br} /$ home/estatistica/populacao/censo2010/default.shtm>

12. Malta DC, Duarte EC. Causas de mortes evitáveis por ações efetivas dos serviços de saúde: uma revisão da literatura. Ciênc Saúde Coletiva. 2007;12(3):765-76.

13. Suba EJ, Donnelly AD, Furia LM, Huynh ML, Raab SS; Viet/American Cervical Cancer Prevention Project. Cervical cancer prevention for all the world's women: genuine promise resides in skilled quality management rather than novel screening approaches. Diagn Cytopathol. 2007;35(3):187-91.

14. Blumenthal PD, Gaffikin L. Cervical cancer prevention: making programs more appropriate and pragmatic. JAMA. 2005;294(17):2225-8.

15. Dias-da-Costa JS, Olinto MTA, Gigante DP, Menezes AMB, Macedo S, Borba AT, et al. Cobertura do exame citopatológico na cidade de Pelotas, Rio Grande do Sul, Brasil. Cad Saúde Pública. 2003; 19(1):191-7.

16. Muller DK, Dias-da-Costa JS, Luz AMH, Olinto MTA. Cobertura do exame citopatológico do colo do útero na cidade de São Leopoldo, Rio Grande do Sul, Brasil. Cad Saúde Pública. 2008;24(11): 2511-20.

17. Moore K, Cofer A, Elliot L, Lanneau G, Walker J, Gold MA. Adolescent cervical dysplasia: histologic evaluation, treatment, and outcomes. Am J Obstet Gynecol. 2007;197(2):141.e 1-6.

18. Deligdisch L, Miranda CRR, Wu HS, Gil J. Human papillomavirusrelated cervical lesions in adolescents: a histologic and morphometric study. Gynecol Oncol. 2003;89(1):52-9.

19. Sousa LB, Pinheiro AK, Barroso MG. Ser mulher portadora do HPV: uma abordagem cultural. Rev Esc Enferm USP. 2008;42(4): 737-43

20. Neumann NA, Tanaka OY, Victoria CG, Cesar JA. Qualidade e equidade da atenção ao pré-natal e ao parto em Criciúma, Santa Catarina, Sul do Brasil. Rev Bras Epidemiol. 2003;6(4):307-18.

21. Agurto I, Bishop A, Sánchez G, Betancourt Z, Robles S. Perceived barriers and benefits to cervical cancer screening in Latin America. Prev Med. 2004;39(1):91-8.

22. Costa JSD, D'Elia PB, Manzolli P, Moreira MR. Cobertura do exame citopatológico na cidade de Pelotas, Brasil. Rev Panam Salud Publica. 1998;3(5):308-13.

23. Hackenhaar AA, Cesar JA, Domingues MR. Exame citopatológico de colo uterino em mulheres com idade entre 20 e 59 anos em Pelotas, RS: prevalência, foco e fatores associados à sua não realização. Rev Bras Epidemiol. 2006;9(1):103-1 1.

24. Dias-da-Costa JS, Madeira ACC, Luz RM, Britto MAP. Auditoria médica: programa de pré-natal em posto de saúde na região Sul do Brasil. Rev Saúde Pública. 2000;34(4):329-36.

25. Bowman JA, Sanson-Fisher RW, Redman S. The accuracy of self-reported Pap smear utilisation. Soc Sci Med. 1997;44(7):969-76. 